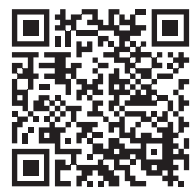

Octubre - Diciembre 2021 Vol. 1, núm. 1 / pp. 26-30

Palabras clave: Anquiloglosia, frenectomía, frenillo lingual.

Keywords: Ankyloglossia, frenectomy, lingual frenulum.

Citar como: Traub VV, Salas BG, Solé VP. Anquiloglosia: una mirada retrospectiva. Lat Am J Oral Maxillofac

Surg. 2021; 1 (1): 26-30. https://dx.doi. org/10.35366/101914

* Cirujano Dentista ${ }^{\ddagger}$ Médico Residente de Cirugía Maxilofacial.

$\S$ Cirujano Maxilofacial.

Universidad de los Andes, Chile.

Recibido: 10/08/2021 Aceptado: 27/08/2021

doi: $10.35366 / 101914$

\title{
Anquiloglosia: una mirada retrospectiva
}

\author{
Ankyloglossia: a retrospective view
}

Vicente Traub Valdés, ${ }^{*}$ Gastón Salas B, ${ }^{\ddagger}$ Pedro Solé V§

\section{RESUMEN}

Objetivo: Analizar de forma retrospectiva la experiencia en anquiloglosia, los profesionales que derivan con mayor frecuencia, edad de diagnóstico, tipo de anquiloglosia más frecuente y técnica utilizada. Material y métodos: Se recopiló la información de fichas clínicas de pacientes diagnosticados con anquiloglosia y luego se realizó un análisis retrospectivo de los datos obtenidos. Resultados: Doscientos siete casos fueron registrados, de los cuales se analizaron distintas variables (sexo, edad, derivador, antecedentes mórbidos, tipo de anquiloglosia, técnica quirúrgica, recidivas y año). Todos los pacientes fueron evaluados por el mismo cirujano, así como todas las cirugías realizadas. Conclusión: E diagnóstico y tratamiento correcto y temprano son imprescindibles para evitar varias consecuencias, desde la restricción del movimiento de la lengua hasta el deterioro del crecimiento mandibular y alteraciones en la fonoarticulación.
\end{abstract}

\section{ABSTRACT}

Objective: To retrospectively analyze the experience in ankyloglossia, the healthcare professionals who refer most frequently, diagnosis age, most frequent tie-tongue type, and surgical technique. Material and methods: Information was collected from clinical records of patient diagnosed with ankyloglossia. A retrospective analysis of the data obtained was carried out. Results: 207 cases were registered, within which a few variables were analyzed (gender, age, referring healthcare professional, morbid history, tied-tongue type, surgical technique, relapse, and year). Every patient was assessed and treated by the same surgeon. Conclusion: Correct and early diagnosis and treatment are essential to avoid many consequences like tongue movement's restriction, mandibular development deterioration and alterations in phonoarticulation.

\section{INTRODUCCIÓN}

La repercusión de la anquiloglosia en la lactancia y en la fonoarticulación es ampliamente discutida y controversial. El diagnóstico y tratamiento quirúrgico han aumentado en las últimas décadas, incluso sin un criterio diagnóstico e indicaciones de tratamiento claros. Un diagnóstico asertivo requiere la completa comprensión del rango anatómico del movimiento de la lengua normal y anormal, vía aérea nasal, labios, tamaño mandibular, función de deglución neonatal, etcétera. ${ }^{1}$

\section{Definición}

De manera tradicional, la anquiloglosia ha sido descrita como la unión del frenillo lingual a la punta de la lengua o cercana a ésta. Recientemente se ha hecho énfasis en un abordaje más funcional con enfoque en los síntomas y signos causados por esta condición. El frenillo lingual, de acuerdo con la International Affiliation of TongueTie Professionals, se define como un remanente de tejido en la línea media entre la cara ventral de la lengua y el piso de la boca. Cuando éste interfiere con la función normal de la lengua se llamará anquiloglosia. ${ }^{1,2}$

No existe una definición estándar de este término. Sin embargo, la anquiloglosia anterior corresponde a la definición previamente mencionada. Esta unión limita la movilidad lingual y protrusión. La inhabilidad de protruir la lengua más allá del bermellón del labio ha sido usada como un hallazgo en el examen clínico indicativo de anquiloglosia. La anquiloglosia posterior es una anquiloglosia sintomática con un frenillo unido en la línea media con el aspecto más posterior de la cara ventral de la lengua. El frenillo puede ser corto, grueso, o incluso submucoso en algunos casos, pero, aun así, puede restringir el movimiento normal de la lengua. Esta 
clasificación de anquiloglosia posterior no está incorporada en el criterio diagnóstico clásico, por lo que aún su diagnóstico es controversial. ${ }^{1}$

Desde el punto de vista anatómico, existen casos sintomáticos en contraposición al hallazgo de un frenillo anatómicamente corto que no se condice con alteración alguna de la función lingual. En el contexto de problemas funcionales que pudiesen comprometer la alimentación, donde el frenillo corto es realmente la única causa, puede ser difícil de determinar. Por ejemplo, se ha visto que el frenillo labial superior corto puede afectar el correcto amamantamiento, y una anquiloglosia presente puede complicar el diagnóstico, asumiéndola como el potencial factor causal. ${ }^{3}$ Otros factores que afectan la lactancia pueden ser la experiencia materna, la producción de leche o incluso la anatomía de las mamas y los pezones. La extensa experticia en cuanto a la deglución y alimentación neonatal por parte de múltiples especialidades, tales como asesoras de lactancia, fonoaudiólogos, matronas y pediatras, ofrece un desafío para el diagnóstico y manejo. La anquiloglosia y la dificultad en la alimentación varía enormemente en cuanto a la severidad de la atadura y de los síntomas asociados. Los clínicos comúnmente deciden tratar la anquiloglosia a pesar del desconocimiento en cuanto a si el cuadro está realmente jugando un rol en la dificultad de la lactancia. ${ }^{1}$

\section{Prevalencia}

La prevalencia de la anquiloglosia en niños ha sido estimada entre 0.1 a $12.11 \%$, lo que refleja la gran variedad de criterios utilizados para el diagnóstico tanto anatómicos como funcionales. ${ }^{4-17}$

\section{Presentación clínica}

La presentación de la anquiloglosia incluye dificultades en el amamantamiento y hallazgos en el examen clínico tanto en el niño como en la madre. En los niños se puede manifestar como un pobre acople o pérdida frecuente de éste, amamantamiento prolongado, irritabilidad, poca ganancia de peso o incapacidad de amamantarse. Al examen clínico puede haber restricción en la movilidad y protrusión lingual, lengua acorazonada. El frenillo puede estar insertado en diferentes niveles a lo largo de la lengua y reborde alveolar. Además, éste puede ser corto, grueso y fibroso pudiendo repercutir aún más sobre la función. El frenillo labial superior restrictivo también ha sido reportado en algunos casos. Por otro lado, las madres pueden experimentar dolor, ulceración y sangramiento de los pezones, baja producción de leche debido a la falta de succión por parte del recién nacido o mastitis. En el caso de la anquiloglosia en niños, adolescentes o adultos la presentación clínica
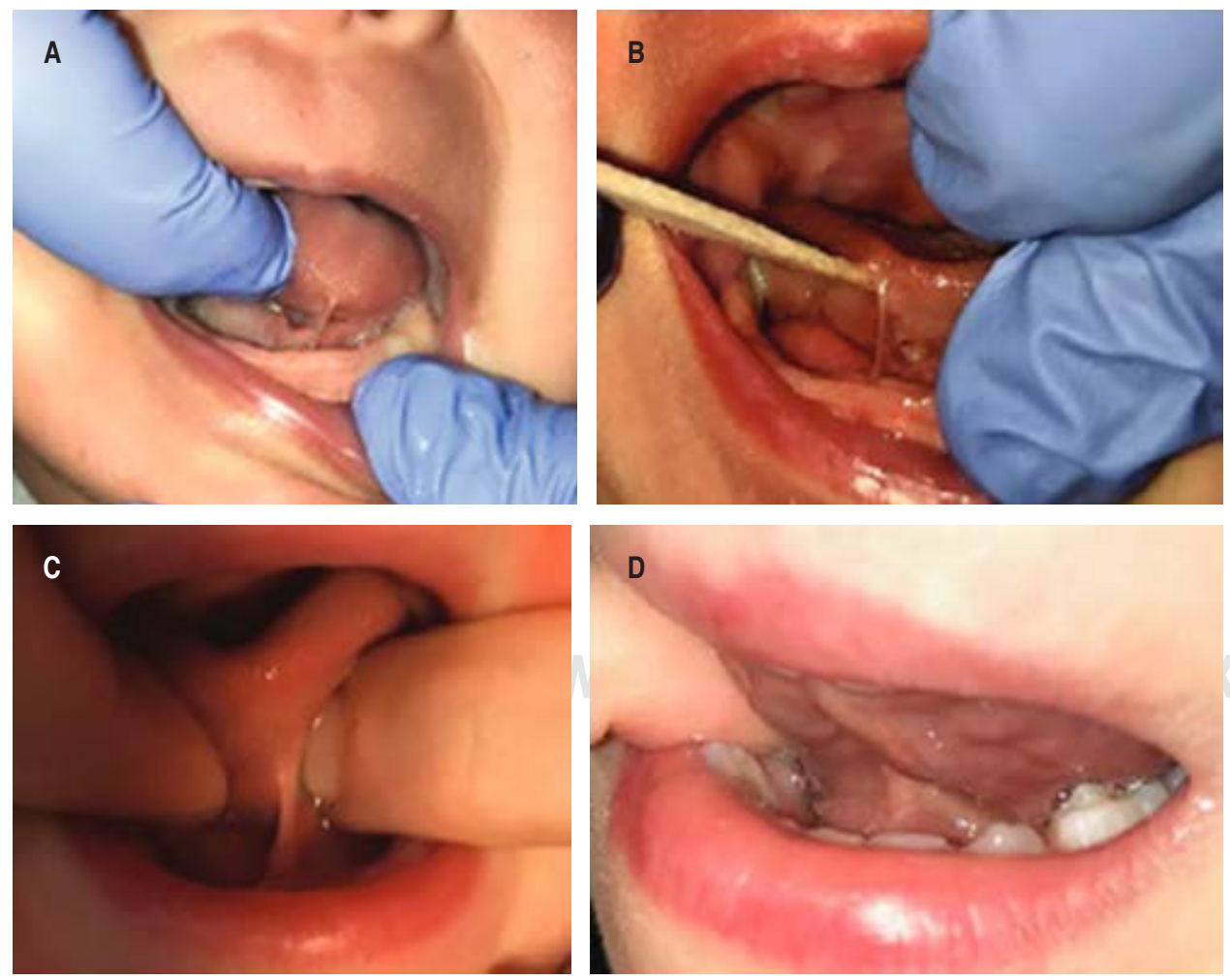

Figura 1:

Clasificación de Coryllos. ${ }^{1}$ A) Tipo I. B) Tipo II. C) Tipo III. D) Tipo IV. 
Tabla 1: Clasificación de Coryllos.

Tipo Descripción

I Frenillo fino y elástico, con inserción en la punta de la lengua, usualmente sobre el reborde alveolar o delante de éste en el surco vestibular

II Frenillo fino y elástico, con inserción de 2-4 mm bajo la punta y sobre o justo tras el reborde alveolar

III Frenillo grueso, con inserción en la mitad de la lengua y mitad del piso de la boca, usualmente menos elástico y más firme

IV Frenillo poco visible, con inserción esencialmente contra la base lingual, grueso, brillante y muy inelástico

será variada, pudiendo presentar trastornos en la deglución, fonoarticulación, desarrollo de los maxilares, trastornos del sueño y ventilatorios, entre otros. ${ }^{1,18,19}$

\section{Clasificación}

Comúnmente las clasificaciones anatómicas toman como referencia el punto de inserción en la lengua, largo del frenillo y protrusión lingual. La clasificación de Coryllos divide la anquiloglosia en cuatro tipos según el punto de inserción en la lengua (Figura 1 y Tabla 1). Los tipos I y II, consideradas las clásicas anquiloglosias, son las más evidentes y comunes probablemente con $75 \%$ de incidencia, mientras que los tipos III y IV son menos comunes, y al ser más difíciles de visualizar son las más subdiagnosticadas y tratadas. El tipo IV es más probable que cause incontinencia y dificultad en la deglución del bolo, lo que provoca más síntomas tanto en el lactante como en la madre. ${ }^{20}$

Otras clasificaciones han incorporado la parte funcional a la anatómica, como la herramienta de evaluación de Hazelbaker (HATLFF), la cual entrega un puntaje tanto para la apariencia del frenillo como para la función lingual, siendo la más completa y compleja guía de asesoramiento para el diagnóstico y tratamiento. ${ }^{21}$ Su uso de forma rutinaria puede ser complejo debido al tiempo que requiere. Por este motivo, se desarrollaron herramientas diagnósticas como la herramienta de evaluación de Bristol (BTAT), que incorpora gran parte de los beneficios de Hazelbaker, pero la hace más fácil y reproducible teniendo una buena correlación con ésta. ${ }^{1,22,23}$

\section{Tratamiento}

El tratamiento primario de la anquiloglosia es la frenectomía. Otros tratamientos no quirúrgicos han sido propuestos por otros profesionales como asesores de lactancia, fonoaudiólogos, etcétera. Las técnicas quirúrgicas incluyen la frenectomía con o sin miotomía, y la Z-plastia. ${ }^{17,24,25}$ En la mayoría de los casos la frenectomía es suficiente. Ésta es clásicamente realizada con un retractor acanalado y una tijera, cortando el frenillo hasta la musculatura lingual. Otras técnicas se han desarrollado con el uso de láser diodo, YAG, Nd YAG, dióxi- do de carbono y electrocauterio, pero que siguen el mismo principio. No hay estudios que demuestren que otra técnica sea mejor que la frenectomía. ${ }^{1}$

El objetivo de este estudio es analizar de forma retrospectiva la experiencia en anquiloglosia, detallando quiénes son los profesionales que derivan con mayor frecuencia, edad de diagnóstico, tipo de anquiloglosia más frecuente y técnica utilizada.

\section{Material y métodos}

Se recopiló la información de 207 fichas clínicas de pacientes diagnosticados con anquiloglosia, derivados por diferentes profesionales de la salud entre enero de 2019 y junio de 2021 al Servicio de Cirugía Maxilofacial del Instituto de Cirugía y Especialidades Odontológicas, Santiago de Chile. Se registró sexo, edad, derivador, antecedentes mórbidos, tipo de anquiloglosia, técnica quirúrgica utilizada, recidivas y año. Todos los pacientes fueron evaluados por el mismo cirujano, así como todas las cirugías realizadas.

\section{Resultados}

Del total de 207 pacientes derivados por anquiloglosia, $40.1 \%$ fue derivado por una asesora de lactancia, 25.6\% por un fonoaudiólogo, $17.4 \%$ por un pediatra, $9.2 \%$ por un odontopediatra u ortodoncista, $4.3 \%$ por una matrona y $3.4 \%$ llegó por consulta espontánea o derivado por otro profesional no registrado.

La edad promedio de los pacientes derivados fue de 14.2 meses (un año dos meses). La mediana se ubica en el rango de un mes, siendo las edades extremas cero meses y 16 años. Veinte punto dos por ciento de los pacientes tienen menos de un mes; $56.2 \%$ se ubica entre uno y seis meses; $3.6 \%$ tiene entre siete y 12 meses; y el $20 \%$ restante se distribuye entre los dos y 16 años.

De los 207 pacientes, sólo 142 frenectomías fueron realizadas (68.6\%). A 11 pacientes se les sugirió mantener una conducta expectante $(5.3 \%)$ y 54 pacientes $(26.1 \%)$ no han sido operados a la fecha por diferentes motivos. Cien por ciento de las frenectomías fueron realizadas con láser diodo y 
divulsión digital. De las cirugías realizadas, sólo 2.8\% fueron a causa de una recidiva, en las cuales se había usado tijeras previamente para la frenectomía. Sólo ocho pacientes (3.8\%) indicaron tener alguna comorbilidad (asma, cardiopatía, dermatitis, displasia de caderas e hipotiroidismo). En cuanto al tipo de anquiloglosia, $10.6 \%$ fue clasificado como tipo I, $26.6 \%$ como tipo II, $2.9 \%$ se clasificó como tipo I-II por tener características de ambos grupos, $22.7 \%$ como tipo III, $6.3 \%$ como tipo II-III, 5.8\% como tipo IV y $4.8 \%$ como tipo III-IV. El $20.3 \%$ restante no tuvo registro en la ficha clínica.

\section{Discusı́ón}

\section{Derivación oportuna}

Debido a los controles regulares que reciben los recién nacidos por parte de distintos profesionales de la salud y sus padres, es posible detectar desde los primeros días de vida alteraciones como la anquiloglosia. Esto se puede ver reflejado en nuestros resultados por el gran porcentaje de pacientes que fueron derivados por asesoras de lactancia (40.1\%), las cuales tienen contacto con el lactante desde los primeros días de vida hasta varios meses más tarde, donde tienen la oportunidad de detectar tempranamente alteraciones en la alimentación del recién nacido. Si a esto se le suman los pacientes derivados por los demás profesionales que tienen contacto temprano (matronas y pediatras), son más de la mitad del total (61.8\%) los que tuvieron la oportunidad de un diagnóstico temprano y derivación oportuna al cirujano maxilofacial para tratar su condición.

Con el transcurso del tiempo, si la anquiloglosia no fue detectada precozmente durante la lactancia, podrá acarrear otros tipos de alteraciones, como por ejemplo dificultad en la fonoarticulación o inclusive anomalías dentomaxilares producto de la restricción de la lengua. Esto llevará a los padres a consultar a un fonoaudiólogo que podrá detectar el problema y derivar. También la erupción de la dentición es un evento en la vida de los niños que tarde o temprano llevará a los padres a consultar a un odontopediatra u ortodoncista, ambos especialistas capacitados para detectar este problema y hacer una derivación oportuna. En adición a lo anteriormente mencionado, otro dato más que apoya esta teoría es la corta edad de los pacientes incluidos, donde $50 \%$ de los pacientes fueron diagnosticados a más tardar al mes de edad.

\section{Conducta}

De un total de 142 cirugías realizadas, en 11 pacientes se mantuvo una conducta expectante, ya que, como indican Walsh y Tunkel ${ }^{1}$ en su revisión, no sólo se debe tener en cuenta la alteración anatómica o apariencia del frenillo, sino que también hay que tener en cuenta si hay o no presencia de una alteración funcional de la lengua. Es por esto que se han desarrollado guías de asesoramiento como la de Hazelbaker ${ }^{21}$ para tomar la mejor decisión para el paciente y no sobre o subtratar.

En cuanto a las cirugías no realizadas los motivos no se explicitan en la ficha clínica, salvo uno en el que la madre no estaba segura de querer realizar el procedimiento a su hijo. El resto se podría asumir que son pacientes que fueron derivados y que realizarán el procedimiento a la brevedad o están en busca de una segunda opinión.

\section{Técnica}

El láser puede ser considerado una alternativa simple y segura para los niños, con reducción del tiempo operatorio, del anestésico local, sangrado, posible infección y malestar. Además, permite una mejor visualización del campo y en la mayoría de los casos elimina la necesidad de sutura. La frenectomía láser permite resultados más predecibles y con menores tasas de recurrencia que la frenectomía convencional. ${ }^{26}$

\section{Tipo}

Según Coryllos ${ }^{20}$ aproximadamente $75 \%$ de la incidencia de las anquiloglosias corresponden al tipo I y II. Según los datos analizados en este estudio, sólo $40.1 \%$ de los casos corresponden al tipo I y II. Sin embargo, esta diferencia podría deberse al $20.3 \%$ de pacientes en los que no se registró el tipo de anquiloglosia. El porcentaje de casos de tipo III y IV corresponde a $28.5 \%$, lo cual se acerca a lo expuesto por Coryllos.

\section{ConClusión}

No hay consenso en cuanto a la indicación quirúrgica real, el tiempo y el tipo de técnica quirúrgica que debe utilizarse.

Se deben considerar tanto aspectos morfológicos como funcionales de la lengua e indicar la cirugía ante limitaciones funcionales si la evaluación demuestra que la función puede mejorar con ésta.

El diagnóstico y tratamiento correcto y temprano son imprescindibles para evitar varias consecuencias, desde la restricción del movimiento de la lengua hasta el deterioro del crecimiento mandibular y alteraciones en la fonoarticulación. En las madres puede causar dificultades en la lactancia que produzcan un destete precoz, alterando la ganancia de peso del lactante.

Es fundamental la formación de una red interdisciplinaria y el trabajo en conjunto de los diferentes profesionales de la salud para el correcto diagnóstico y tratamiento de la anquiloglosia. 


\section{RefERENCIAS}

1. Walsh J, Tunkel D. Diagnosis and treatment of ankyloglossia in newborns and infants: a review. JAMA Otolaryngol Head Neck Surg. 2017; 143 (10): 1032-1039.

2. Tongue Tie Professionals-IATP [Internet]. [Citado 9 de agosto de 2021] Disponible en: https://tonguetieprofessionals.org/

3. Ghaheri BA, Cole M, Fausel SC, Chuop M, Mace JC. Breastfeeding improvement following tongue-tie and lip-tie release: a prospective cohort study. Laryngoscope. 2017; 127 (5): 1217-1223.

4. Catlin FI. Tongue-tie. Arch Otolaryngol. 1971; 94 (6): 548-557.

5. Jorgenson RJ, Shapiro SD, Salinas CF, Levin LS. Intraoral findings and anomalies in neonates. Pediatrics. 1982; 69 (5): 577-582.

6. Flinck A, Paludan A, Matsson L, Holm AK, Axelsson I. Oral findings in a group of newborn Swedish children. Int J Paediatr Dent. 1994; 4 (2): 67-73.

7. González Jiménez D, Costa Romero M, Riaño Galán I, González Martínez MT, Rodríguez Pando MC, Lobete Prieto C. Prevalence of ankyloglossia in newborns in Asturias (Spain). An Pediatr (Barc). 2014; 81 (2): 115-119.

8. García Pola MJ, González García M, García Martín JM, Gallas M, Seoane Lestón J. A study of pathology associated with short lingual frenum. ASDC J Dent Child. 2002; 69 (1): 59-62.

9. Sedano HO. Congenital oral anomalies in Argentinian children. Community Dent Oral Epidemiol. 1975; 3 (2): 61-63.

10. Sawyer DR, Taiwo EO, Mosadomi A. Oral anomalies in Nigerian children. Community Dent Oral Epidemiol. 1984; 12 (4): 269-273.

11. Salem G, Holm SA, Fattah R, Basset S, Nasser C. Developmental oral anomalies among schoolchildren in Gizan region, Saudi Arabia. Community Dent Oral Epidemiol. 1987; 15 (3): 150-151.

12. Voros-Balog T, Vincze N, Bánóczy J. Prevalence of tongue lesions in Hungarian children. Oral Dis. 2003; 9 (2): 84-87.

13. Mumcu G, Cimilli H, Sur H, Hayran O, Atalay T. Prevalence and distribution of oral lesions: a cross-sectional study in Turkey. Oral Dis. 2005; 11 (2): 81-87.

14. Ugar-Cankal D, Denizci S, Hocaoglu T. Prevalence of tongue lesions among Turkish schoolchildren. Saudi Med J. 2005; 26 (12): 1962-1967.

15. Freudenberger S, Santos Díaz MA, Bravo JM, Sedano HO. Intraoral findings and other developmental conditions in Mexican neonates. J Dent Child (Chic). 2008; 75 (3): 280-286.
16. Cinar F, Onat N. Prevalence and consequences of a forgotten entity: ankyloglossia. Plast Reconstr Surg. 2005; 115 (1): 355-356.

17. Lalakea ML, Messner AH. Ankyloglossia: does it matter? Pediatr Clin North Am. 2003; 50 (2): 381-397.

18. Kotlow L. Diagnosis and treatment of ankyloglossia and tied maxillary fraenum in infants using Er:YAG and 1064 diode lasers. Eur Arch Paediatr Dent. 2011; 12 (2): 106-112.

19. Pompéia LE, Ilinsky RS, Ortolani CLF, Faltin K. Ankyloglossia and its influence on growth and development of the stomatognatic system. Rev Paul Pediatr. 2017; 35 (2): 216-221.

20. Corrylos E, Genna CW, Salloum AC. Congenital tongue-tie and its impact on breastfeeding. AAP Section on Breastfeeding Bulletin, summer 2004.

21. Hazelbaker A, Baeza C, Genna C, Murphy J, Kaplan M, Martinelli R, et al. Assessment and classification of tongue-tie. Clinical Lactation. 2017; 8: 93-98.

22. Madlon-Kay DJ, Ricke LA, Baker NJ, DeFor TA. Case series of 148 tongue-tied newborn babies evaluated with the assessment tool for lingual frenulum function. Midwifery. 2008; 24 (3): 353-357.

23. Ingram J, Johnson D, Copeland M, Churchill C, Taylor H, Emond A. The development of a tongue assessment tool to assist with tonguetie identification. Arch Dis Child Fetal Neonatal Ed. 2015; 100 (4): F344-F348.

24. Junqueira MA, Cunha NN, Costa e Silva LL, Araújo LB, Moretti $A B$, Couto Filho CE, et al. Surgical techniques for the treatment of ankyloglossia in children: a case series. J Appl Oral Sci. 2014; 22 (3): 241-248.

25. Horton CE, Crawford HH, Adamson JE, Ashbell TS. Tongue-tie. Cleft Palate J. 1969; 6: 8-23.

26. Azevedo M, Oliveira N, Lucas L, Borges L, Silveira A, Gomes C, et al. Técnicas quirúrgicas para el tratamiento de la anquiloglosia en niños: serie de casos. Appl Oral Sci. 2014; 22 (3): 241-248.

Correspondencia:

Dr. Vicente Traub Valdés

E-mail: vatraub@miuandes.cl 